\title{
Modelo de conocimiento conceptual como apoyo a la Ingeniería del Conocimiento
}

\section{Conceptual knowledge modeling as support in knowledge engineering}

\author{
Mónica Henao-Calad ${ }^{1} \quad$ Vanessa Rodríguez-Lora ${ }^{2}$ \\ Recibido 22 de marzo de 2012, aceptado 13 de agosto de 2012 \\ Received: March 22, 2012 Accepted: August 13, 2012
}

\begin{abstract}
RESUMEN
Este artículo presenta una propuesta del uso de modelos de conocimientos para apoyar los procesos de Ingeniería del Conocimiento. El modelo de conocimiento propuesto permite representar el conocimiento declarativo de un dominio de una manera estructurada, a través de la técnica de representación de conocimiento de mapas conceptuales.

La Ingeniería del Conocimiento se ha relacionado con el desarrollo de sistemas basados en el conocimiento en el cual el conocimiento y el razonamiento juegan un papel primordial. Como disciplina, dirige la tarea de construir sistemas inteligentes proporcionando herramientas y métodos que soportan el desarrollo de estos sistemas, involucrando: obtención de conocimiento, familiarización con el dominio, análisis y el diseño de la solución, y validación del conocimiento. Todo esto integra los procesos de la Ingeniería del Conocimiento: adquisición, representación y manipulación / validación del conocimiento.

En este artículo se muestra cómo un modelo de conocimientos basado en mapas conceptuales apoya la realización de cada uno de esos procesos, permitiendo que los conceptos del dominio sean fácilmente identificados y jerarquizados, logrando generar una visión global del dominio y una homologación de sus conceptos. Adicionalmente, se presenta el caso de un modelo de conocimiento desarrollado por el grupo de la Gerencia en Colombia de la Universidad EAFIT ${ }^{3}$ como parte del proyecto "Petroleum Geology of Colombia" que se realizó en el Departamento de Geología de esta universidad y patrocinado por la Agencia Nacional de Hidrocarburos - $\mathrm{ANH}^{4}$ de Colombia, en el que se representó el conocimiento de geólogos e ingenieros expertos en cuencas petrolíferas colombianas.
\end{abstract}

Palabras clave: Ingeniería del conocimiento, modelo de conocimientos, CmapTools, representación de conocimiento, adquisición de conocimiento, manipulación de conocimiento.

\begin{abstract}
This article introduces a proposal for knowledge models use to support knowledge engineering processes. The proposed knowledge model allows the presentation on a structured way of a domain's declarative knowledge, through the knowledge representation technique of concept maps.

Knowledge Engineering has been related with the development of systems based on knowledge where knowledge and reasoning are the main players. As a discipline, leads the task of building smart systems offering tools and methods that support the implementation of such system, involving: knowledge gathering, getting to know the domain, solution analysis and design, and knowledge validation. All of this integrates the knowledge engineering processes: acquisition, representation and knowledge manipulation/validation. In this article shows how a knowledge model based on concept maps supports the achievement of each of these processes, allowing domain concepts to be easily identifiable and organized on a hierarchical way,
\end{abstract}

\footnotetext{
1 Departamento de Organización y Gerencia. Universidad EAFIT. Medellín, Colombia. E-mail: mhenao@eafit.edu.co

2 Departamento de Informática y Sistemas. Universidad EAFIT. Medellín, Colombia. E-mail: 1rodrig5@eafit.edu.co

3 www.eafit.edu.co

4 http://www.anh.gov.co
} 
achieving the generation of a global domain vision and a homologation of its concepts. Additionally, the case of a knowledge model is presented, created as part of the project "Petroleum Geology of Colombia" being implemented on the Geology department of EAFIT University, and sponsored by ANH of Colombia, where the knowledge of geologist and engineers experts on Colombian petroliferous basins is presented.

Keywords: Knowledge engineering, knowledge model, CmapTools, knowledge representation, knowledge acquisition, knowledge manipulation.

\section{INTRODUCCIÓN}

Este artículo presenta la estrategia de Modelo de Conocimientos que permite representar el conocimiento de un dominio de una manera estructurada, identificándolo y describiéndolo de una manera gráfica, lo que posibilita tener una vista holística e integrada de su contenido a través de objetos de conocimiento.

Para llevar a cabo el modelado del conocimiento es necesario realizar los procesos de elicitación y representación de dicho conocimiento. El primero se refiere a todas las actividades y técnicas que son necesarias de aplicar para poder extraer el conocimiento del dominio de las fuentes que lo contienen, bien sea fuentes dinámicas como los expertos o fuentes estáticas como los libros. El segundo se refiere a las actividades y técnicas para representar el conocimiento extraído de las fuentes, con el objetivo de tener un modelo ajustado al conocimiento original. Dichos procesos hacen parte de la Ingeniería del conocimiento, área fundamental de la Inteligencia Artificial y cuyo principal objetivo es poder tener modelos de conocimiento que puedan llegar a ser incluso automatizados en un computador.

Este artículo incluye entonces aspectos teóricos de modelos de conocimiento, de Ingeniería del Conocimiento y en especial de representación del conocimiento.

Además, se presentan algunos apartes importantes de un modelo de conocimientos que se desarrolló para representar el conocimiento declarativo de las cuencas petrolíferas colombianas. Este modelo hace parte de un proyecto que se está realizando en el Departamento de Geología de la Universidad EAFIT y que es patrocinado por la Agencia Nacional de Hidrocarburos-ANH de Colombia.
Por último, se plantean las conclusiones y las referencias utilizadas en este artículo.

\section{PILARES CONCEPTUALES DEL MODELO DE CONOCIMIENTOS}

\section{Qué es un modelo de conocimientos}

Dado que un modelo es una vista simplificada de una realidad compleja, implicando la creación de una abstracción que permite entender mejor la realidad del dominio [1], entonces un modelo de conocimiento identifica y describe el conocimiento de un dominio particular, dando una vista holística e integrada del contenido del conocimiento [2].

El modelo de conocimiento es una imagen del conocimiento y se compone de elementos primitivos llamados objetos de conocimiento que pueden ser de varios tipos ${ }^{5}$ :

- Conceptos: Los conceptos son cosas que constituyen un dominio. Pueden ser objetos físicos, cosas abstractas, personas, entre otras. Cada concepto se describe por las relaciones que tiene con otros conceptos en el dominio, por ejemplo a través de una jerarquía, y por sus atributos y valores. Desde la perspectiva gramatical, los conceptos son usualmente equivalentes a sustantivos. Por ejemplo: cuenca petrolífera, pozo, petróleo.

- Instancias: Las instancias son ejemplos particulares de una clase o concepto y por eso sólo tienen atributos de esa clase. Por ejemplo: Cuenca del Chocó, Pozo Buchadó.

- Procesos (tareas y actividades): Son una serie de acciones ejecutadas para satisfacer una meta o una serie de objetivos. Son una secuencia de

5 http://www.epistemics.co.uk/Notes/87-0-0.htm 
estados y eventos [3]. Por ejemplo: Explotación de petróleo.

- Atributos y valores: Describen las propiedades de los objetos de conocimiento. Los atributos son las propiedades genéricas, cualificaciones o características que pertenecen a una clase de conceptos. Por ejemplo, profundidad, calidad, extensión. Los valores son las cualidades específicas de un concepto, tal como el volumen actual de un pozo. Los valores están asociados con un atributo particular y pueden ser numéricos (por ejemplo $15539 \mathrm{ft}$.) o de categorías (grande, productivo). Desde una perspectiva gramatical un valor es equivalente a un adjetivo.

- Reglas: Son instrucciones de la forma SI... ENTONCES... Por ejemplo: SI el conocimiento es conceptual ENTONCES hacer un mapa conceptual.

- Relaciones: Representan la forma en que los objetos de conocimiento (tales como conceptos y tareas) están relacionados con otros. Por ejemplo la relación ES-A para mostrar la clasificación, PARTE-DE para mostrar composición. Las relaciones son a menudo representadas como flechas o diagramas. Desde una perspectiva gramatical las relaciones son usualmente equivalentes a verbos pasivos.

Además, esos objetos de conocimiento son de diferente tipo dependiendo del conocimiento que se representa. A continuación se presenta una clasificación muy común del conocimiento:

- Conocimiento declarativo, procedimental o argumentativo.

- El conocimiento declarativo es el conocimiento de los hechos o también llamado "saber qué". Este conocimiento es consciente y puede ser verbalizado (escrito, hablado, pintado, entre otras) [4]. Comúnmente se denomina "conocimiento conceptual". Por ejemplo: la definición de un modelo de conocimientos.

- El conocimiento procedimental es el conocimiento de cómo hacer las cosas o llamado también "saber cómo". Un procedimiento, como [3] lo define, es un patrón que determina los tipos de estados y eventos que pueden ocurrir en un proceso. Puede ser difícil de explicar pues está asociado con las habilidades y la experiencia. Por ejemplo, diseñar un modelo de conocimientos sobre cuencas petrolíferas.

- El conocimiento argumentativo es el conocimiento del "saber-por qué" y se refiere al conocimiento que direcciona la noción de la razón o significado de algún fenómeno. "Éste orienta y define las acciones humanas y se relaciona con el sistema de valores propagado por las instituciones de la sociedad" [4].

- Conocimiento tácito o explícito. El tácito no puede ser articulado fácilmente y se refiere al conocimiento que es propio del ser humano y que se basa en su experiencia, creencias, perspectivas, entre otras. El conocimiento explícito es el que está formalizado en un lenguaje y que puede ser expresado por la persona de alguna forma.

- Conocimiento genérico o específico. El conocimiento genérico se puede aplicar a muchas situaciones y el específico a una o a pocas situaciones.

Entonces, cuando se habla de modelo de conocimiento se debe determinar qué tipo de conocimientos es el que se va a representar (declarativo o procedimental). De esta forma surgen dos tipos generales de modelos de conocimiento:

- Modelo de conocimiento conceptual. Es una representación gráfica del conocimiento conceptual de un dominio del mundo real [5]. Es la representación de objetos y eventos y cómo ellos están relacionados con otros objetos y eventos. Por tanto se enfoca en el qué y el por qué más que en el cómo. Puede ser usado para facilitar el diseño e implementación de un sistema de información o como herramienta para la puesta en común y la definición estándar de un conocimiento conceptual correcto y apropiado para un dominio en particular.

- Modelo de conocimiento procedimental. Es una representación gráfica del conocimiento 
procedimental de un dominio. Es la representación de las tareas o pasos que se requieren realizar para alcanzar un objetivo.

Además, es importante reconocer si el conocimiento a representar en el modelo hace parte del conocimiento tácito o explícito de una persona, y si es genérico a específico. Lo primero es necesario para identificar las fuentes del conocimiento y determinar las mejores técnicas para su elicitación. Y lo segundo, para saber si es posible hacer abstracciones del conocimiento que permitan aplicarse en diferentes situaciones.

Pero, independiente del tipo de conocimiento que se esté modelando, el modelo debe representar el dominio con exactitud y completitud.

\section{Para qué se hace un modelo de conocimientos}

Como lo plantean [2], la toma de decisiones es uno de los procesos fundamentales en un negocio o en un proyecto, en donde se plantean preguntas como: ¿Cuál es la mejor solución a un problema en particular? ¿Cómo hacer para conservar un conocimiento en particular? ¿Cuándo se debe hacer un modelo de conocimiento? ¿Cómo se representa el conocimiento?, entre otras, pues esa lista puede llegar a ser interminable. Quienes se enfrentan a esas preguntas y deben decidir qué hacer, deben tomar decisiones que están limitadas por la disponibilidad del conocimiento. Por tanto, tener el conocimiento representado y organizado es fundamental para apoyar dicho proceso de toma de decisiones. De esta forma el conocimiento estaría disponible, lo que podría ayudar a mejorar el desempeño individual y organizacional.

Por otro lado, el modelo de conocimientos sirve para estructurar proyectos relacionados con el dominio del modelo, para adquirir y validar los conocimientos representados en él y para almacenar dicho conocimiento para uso futuro.

Como lo plantea Gregory Judelman [4] en su tesis "Knowledge Visualization; Problems and Principles for zapping the Knowledge Space", "A la fecha hay una enorme cantidad de conocimiento experto producido y documentado, pero muchas veces el conocimiento necesario no está disponible en el lugar correcto en el momento correcto para ser puesto en acción. Culturas pasadas, en particular las predigitales, pueden haber tenido problema con el acceso a la información, pero hoy en día la cantidad de información libre que está disponible sólo en Internet es enorme. La restricción no es el acceso sino filtrar, hallar y evaluar la calidad del contenido de la información".

Debido a esto, han surgido con más fuerza los modelos de conocimiento que posibilitan la definición del espacio de conocimiento de un dominio en particular, en un lenguaje correcto, accediendo incluso a recursos digitales relevantes para el conocimiento.

\section{INGENIERÍA DEL CONOCIMIENTO (IC)}

La IC es una disciplina derivada de la Inteligencia Artificial, que tiene como propósito definir técnicas, modelos y metodologías para adquirir, representar y manipular el conocimiento de un dominio y que pueda ser llevado a un Sistema Basado en el Conocimiento (SBC). Un SBC deberá seguir una lógica de operación o heurística similar a la que utiliza el experto humano en un dominio en particular para la solución de un problema. La tarea de adquirir, modelar y manipular el conocimiento es función del Ingeniero del conocimiento [6], quien conoce y domina lo que se propone en la Ingeniería del Conocimiento.

La IC ha definido un proceso en el que se busca rescatar el conocimiento desestructurado de los expertos a través de diferentes actividades, entre ellas entrevistas, para posteriormente codificarlo y utilizarlo [7]. Este proceso tiene tres actividades que son claves para manejar el conocimiento: la adquisición, representación y manipulación de conocimiento (ver Figura 1).

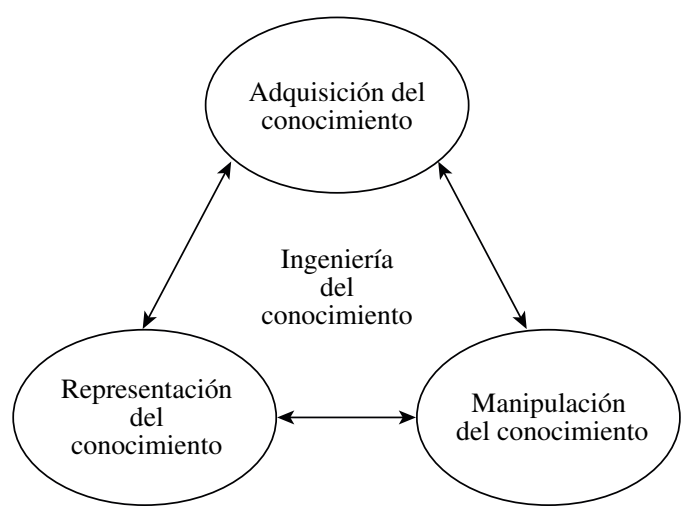

Figura 1. Procesos de la Ingeniería del Conocimiento (creación propia). 


\section{Adquisición del conocimiento}

La adquisición de conocimiento es el proceso inicial de la Ingeniería del Conocimiento y se divide en dos fases:

- La elicitación de conocimiento que se hace a través de las actividades de extracción y educción [8].

- En la extracción, el conocimiento proviene de fuentes estáticas, es decir de repositorios de conocimiento que por sí mismos no pueden cambiar el conocimiento allí contenido, como por ejemplo un libro. Se utilizan técnicas como el análisis de documentos y el estudio de casos, entre otras.

- La educción de conocimiento se concentra en la captura del conocimiento que poseen las fuentes dinámicas o expertos, tomando su conocimiento tácito y explícito para que pueda ser asequible y pueda quedar en un formato explícito, ordenado y fácil de entender y que pueda ser usado por otros [8]. Entre las técnicas que se utilizan para esto están el análisis de protocolos, las entrevistas y las críticas, entre otras.

- La validación del conocimiento se realiza para verificar que el conocimiento elicitado es correcto y apropiado. Entre las técnicas que se utilizan para esto están el prototipado, teach back y la simulación, entre otras.

La adquisición es tradicionalmente considerada como una de las actividades más complejas dentro de la IC [9], ya que en su desarrollo deben considerarse un número importante de variables que podrían afectar el producto de esta fase. Esta actividad debe desarrollarse de la forma más eficiente posible, haciendo una buena planeación que permita maximizar los resultados obtenidos y usando la menor cantidad de recursos [10]. Para ello deben definirse metodologías, modelos, técnicas, que permitan enfocarse en el conocimiento que se necesita.

\section{Representación del conocimiento}

En [11] se define la representación como un conjunto de convenciones sintácticas y semánticas que hacen posible la descripción de las cosas y que además permite realizar razonamientos sobre el dominio representado empleando en ello el lenguaje como medio de expresión humana [14].

Por sintaxis se define al conjunto de reglas que combinadas con un conjunto de símbolos permite validar expresiones del lenguaje; hace alusión a la forma, al cómo. La semántica es la especificación del cómo esas expresiones se interpretan, qué significado tienen; se relaciona con el qué [12].

Además, se debe buscar que la representación sea completa, concisa, coherente, concreta, conceptual, correcta y considerada [13].

Por tanto, el sistema de representación debe tener dos características fundamentales: la primera se relaciona con su poder expresivo, es decir, con la facilidad de la descripción; la segunda se relaciona con la forma que tiene para eliminar o evitar las ambigüedades [12].

Sin embargo, las representaciones como tal no son perfectas, son simplemente aproximaciones de la realidad [14] y como consecuencia pueden introducir errores que conllevan a malas conclusiones sobre el dominio, sin que ello signifique que el razonamiento o la inferencia sean erróneos. Por ello es necesario en el proceso de validación hacer una revisión exhaustiva del conocimiento representado para disminuir el margen de error.

Existen muchas técnicas para representar el conocimiento, así como existen diferentes factores que ayudan a determinar la selección de una en particular, por ejemplo dependiendo del tipo de conocimiento que se quiere representar. Es tarea del ingeniero del conocimiento seleccionar las técnicas adecuadas para representar el conocimiento que ya se ha adquirido, esto para evitar que las diferentes representaciones afecten la manera en la que el conocimiento pueda ser asimilado [15]. Entre las técnicas más empleadas para la representación de conocimiento se encuentran las reglas de producción, las redes semánticas, los mapas conceptuales, los mapas mentales y los diagramas de flujo.

\section{Manipulación de conocimiento}

La manipulación del conocimiento tiene como finalidad la validación del conocimiento representado 
y la verificación del funcionamiento del modelo desarrollado [6]. En este proceso participa tanto el ingeniero del conocimiento como el experto y los usuarios del modelo, cada uno verificando aspectos particulares del modelo. Las observaciones que resultan en este proceso implican realizar de nuevo actividades relacionadas con la adquisición y la representación del conocimiento. Se da por terminado el proceso cuando el modelo es aprobado y acogido para comenzar su vida útil real.

\section{PROPUESTA DE LO QUE DEBE SER UN MODELO DE CONOCIMIENTO BASADO EN MAPAS CONCEPTUALES COMO APOYO A LA INGENIERÍA DEL CONOCIMIENTO}

El esquema de modelado de conocimiento basado en mapas conceptuales fue desarrollado en el Institute for Human and Machine Cognition (IHMC) ${ }^{6}$ en Estados Unidos. En [16-18] se argumenta que "el modelado del conocimiento comienza con una creación autónoma o elicitación de una serie de mapas conceptuales interrelacionados e interconectados pertenecientes al dominio de conocimiento. Esta colección de mapas conceptuales contiene y cubre el conocimiento conceptual del dominio y sirve como un mecanismo de articulación de otros recursos electrónicos relacionados con los conceptos de los mapas".

También investigadores del IHMC en conjunto con investigadores de NASA Glenn Research Center ${ }^{7}$ propusieron un método para modelado de conocimientos llamado PreSERVe (Prepare, Scope, Elicit, Render, Verify) [17] que involucra un proceso de preparación para la elicitación del conocimiento, seguido por un proceso iterativo de evaluación del alcance, elicitación e interpretación de conocimiento por varios métodos, y verificación con los expertos.

Los mapas conceptuales representan el conocimiento conceptual de un dominio. Por tanto, son una representación explícita y concisa tanto de lo que es el conocimiento explícito del dominio como de lo que el experto sabe, posibilitando que dicho experto defina apropiadamente los límites de su dominio

\footnotetext{
6 http://www.ihmc.us

7 http://www.nasa.gov/centers/glenn/home/index.ht ml
}

de conocimiento y facilitando la verificación del conocimiento representado.

Estos mapas son gráficos que se componen de conceptos (definido por Novak y Gowin en [19] como regularidades percibidas en objetos y eventos) en los nodos y las relaciones entre los conceptos en los arcos. Los mapas conceptuales son usados para formar modelos de conocimiento basados en una organización jerárquica y con unos medios que pueden ser adicionados a cada nodo del mapa. Todo el modelo completo se encadena a través de un mapa general, subsumiendo de nivel superior. El modelo resultado del conocimiento del experto contiene numerosos (cientos) de conceptos del dominio, principios y relaciones que son extraídas del experto, filtrados y verificados usando el método PreSERVe.

A diferencia de los enlaces en los navegadores de Internet que transmiten ningún contenido semántico, las conexiones entre los conceptos en los mapas conceptuales tienen un significado explícito en dos formas distintas: 1) Los vínculos entre conceptos dentro de un solo mapa elaboran las relaciones entre los conceptos en ese mapa; 2) El mecanismo de enlace permite que un concepto en un mapa conceptual pueda ser vinculado con el mismo concepto en otro mapa, lo que posibilita tener elaboraciones más generales o más específicas del concepto y de examinar el concepto en diferentes contextos. Y hay una función de búsqueda que permite que el usuario del modelo de conocimiento identifique lugares en el modelo que contienen información sobre temas de interés.

Los modelos de conocimiento de este tipo contienen contenido que es diferente de la información más general que se encuentra en los materiales típicos de referencia y que se organiza de manera muy diferente al texto o sistema de aprendizaje hipermedia estándar (secuencial). Estos modelos de conocimiento tienden a ser grandes y complejos (lo que refleja la complejidad de los dominios reales de conocimiento) con temas entrelazados y ricas interconexiones de los conceptos basados en el modelo mental altamente articulado de los expertos del dominio. Como tal, estos modelos de conocimiento contienen el conocimiento más específico del dominio y que es más directamente aplicable a las situaciones problemáticas específicas. 
Utilizar la técnica de mapas conceptuales y las pautas de los procesos de IC para la creación de modelos de conocimiento, facilita y asegura que el conocimiento final represente adecuadamente el conocimiento original. Así, cuando se realiza el proceso de obtención del conocimiento se utilizan los mapas como una herramienta que permite que los expertos puedan explicitar su conocimiento más fácilmente y puedan confirmar el conocimiento que ellos tienen. Esto conduce incluso a la creación de artefactos (mapas conceptuales, transcripciones de entrevistas, videos editados de los expertos discutiendo un tema o hacer un punto, entre otros) que se pueden incluir en el modelo de conocimiento.

Adicionalmente, en el proceso de adquisición del conocimiento el modelo de conocimientos desarrollado se puede emplear para identificar nuevo conocimiento o conceptos que no se han identificado previamente o que no se han relacionado con conceptos ya existentes dentro del mismo modelo. La Figura 2 muestra como el modelo de conocimientos puede ser empleado para adquirir e identificar nuevos conceptos.

Como se puede observar, al mismo tiempo que se hace la adquisición del conocimiento se está

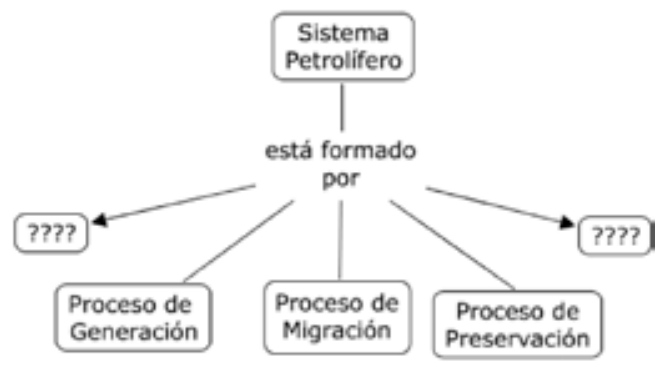

Figura 2. Mapa empleado para obtener nuevos conceptos en el tema de cuencas Petrolíferas Colombianas (creación propia).

haciendo la representación del mismo. Por tanto, se está realizando el proceso de representar el conocimiento a través de mapas conceptuales que integrarán el modelo del conocimiento conceptual. Esto permitirá establecer jerarquías y relaciones entre los conceptos propios del dominio, facilitando la identificación de categorías y atributos propios de los conceptos y permitiendo ver de una forma global las relaciones entre ellos. En la Figura 3 se muestran las diferentes cuencas de petróleos que se describen en el modelo realizado en el marco del proyecto de la $\mathrm{ANH}$.

\section{ANH}

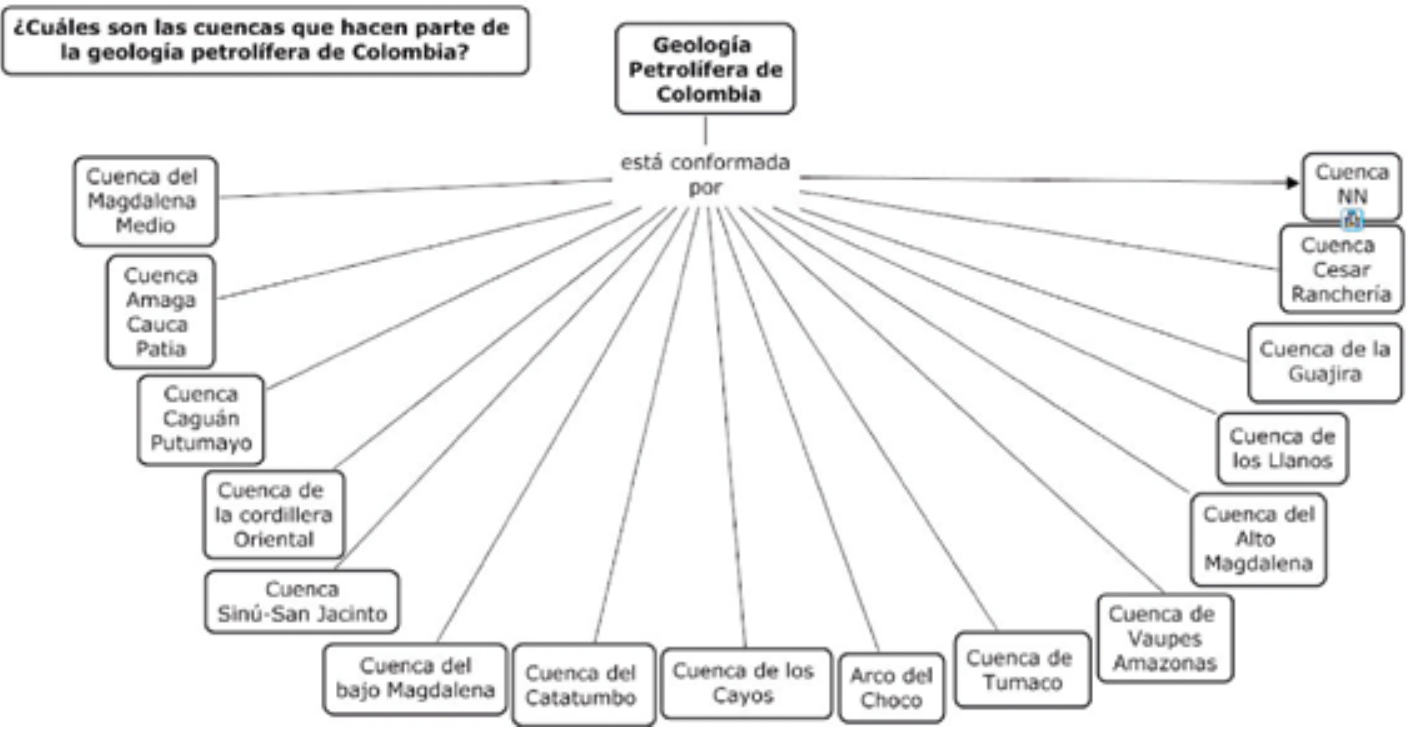

Figura 3. Mapa principal, Petroleum Geology of Colombia [20]. 
En el proceso de manipulación de conocimiento estos modelos facilitan la validación de los conceptos y sus relaciones, lo cual es muy importante para asegurar el conocimiento representado. Es necesario entonces hacer esta revisión para cada uno de los mapas con el propósito de garantizar que el conocimiento que esté allí consignado sea el que el experto considera que es el correcto para el dominio en particular. Las siguientes imágenes (Figuras 4 y 5) corresponden a diferentes versiones del mismo mapa, que junto con los expertos pudo refinarse, de tal modo que fuera más claro para ellos.

\section{EJEMPLO DE LA APLICACIÓN DE INGENIERÍA DEL CONOCIMIENTO EN EL CAMPO DE CUENCAS PETROLÍFERAS}

El departamento de Geología de la Universidad EAFIT desarrolló el proyecto "Petroleum geology of Colombia" para la Agencia Nacional de HidrocarburosANH, en el cual se buscaba hacer una síntesis del conocimiento que se tenía sobre la geología del petróleo de Colombia, mostrando para ello los aspectos geológicos que se identificaron en las

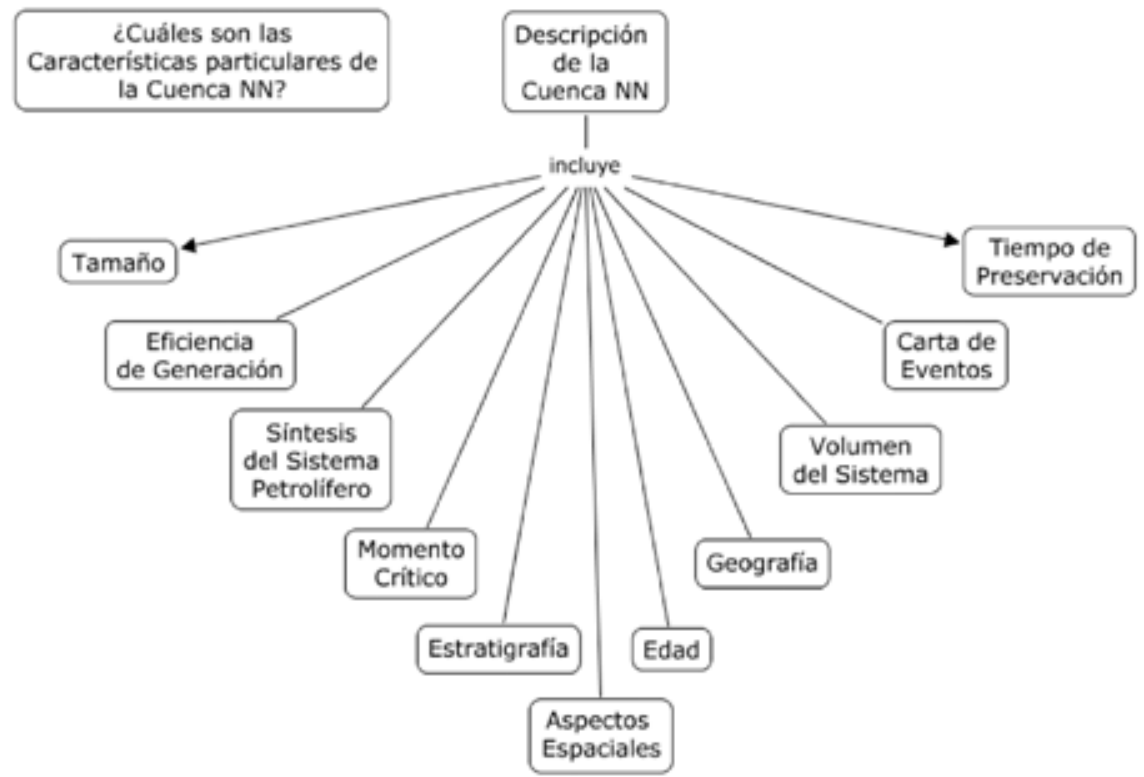

Figura 4. Mapa conceptual de descripción de cuenca antes del proceso de validación [20].

\section{ANH \\ ANH \\ COLOLBIA}

¿Cuáles son las características

de la cuenca NN?

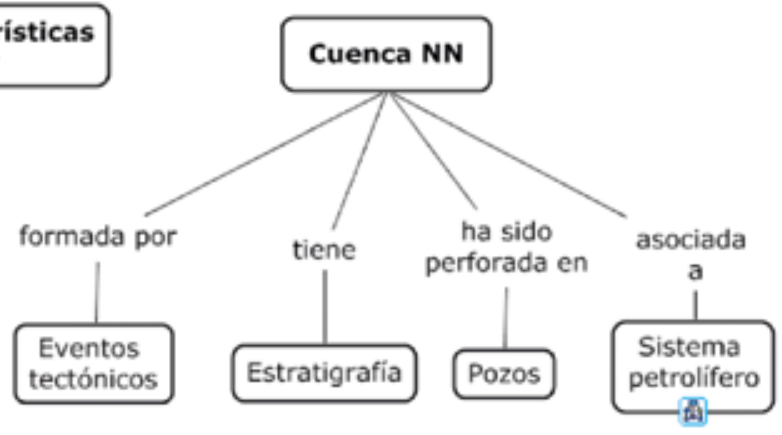

Figura 5. Mapa conceptual de descripción de cuenca después del proceso de validación [20]. 
exploraciones de diferentes cuencas de hidrocarburos durante más de 70 años. Como producto principal de este proyecto se tienen 15 libros donde está compilada esta información. Cada uno de estos libros es el resultado del esfuerzo conjunto de diferentes centros de investigación y hace parte de la memoria de conocimiento que se tiene al respecto.

Pero, dado que el desarrollo de un libro tradicional exige un orden secuencial y está estructurado de un modo que no permite "evidenciar" las relaciones entre los conocimientos presentados, se vio la necesidad de complementar el proyecto, presentando el conocimiento en una forma diferente, para que fácilmente se pudiera tener una visión global o completa del dominio y pudiera ser comprendido por personas que no son expertas en él. Resultando así en la creación del modelo de conocimientos de cuencas petrolíferas colombianas, que a continuación se presenta.

\section{Modelo de conocimiento de cuencas petrolíferas colombianas}

Para apoyar el proyecto de los 15 libros, se diseñó un modelo de conocimientos usando la estrategia de mapas conceptuales. Este modelo fue desarrollado por integrantes del grupo de investigación de la Gerencia en Colombia ${ }^{8}$.

Se utilizó la herramienta CmapTools ${ }^{9}$, software especializado para hacer este tipo de representación. CmapTools permite generar modelos de este tipo a gran escala (es decir, modelos que contienen decenas de mapas conceptuales, cientos de proposiciones y de recursos multimedia) y luego usar esos modelos para integrar y navegar a través de los recursos electrónicos asociados a los conceptos e incluso ponerlos a disposición a través de un portal en Internet [21]. De esa forma se pueden tener documentos de texto, gráficos, archivos de audio o video, enlaces a páginas Web y otros tipos de documentos asociados con el dominio de las cuencas colombianas.

El modelo desarrollado para el proyecto presenta los conceptos que hacen parte de la descripción general de las cuencas de petróleo y los elementos que constituyen su correspondiente sistema petrolífero.

\footnotetext{
8 http://201.234.78.173:8080/gruplac/jsp/visualiza/visualizagr. jsp?nro $=00000000001632$

9 http://cmap.ihmc.us/
}

Para realizar la extracción y representación del conocimiento de las cuencas se decidió seguir las pautas y los procesos propuestos en la IC. Inicialmente se identificaron las fuentes de conocimiento del dominio. Primero a los expertos quienes tienen conocimiento muy valioso y especial en el tema. Se trabajó principalmente con el doctor Fabio Cediel Melo, quien se desempeñó como coordinador de investigación del proyecto y quien es además reconocido como experto nacional en el tema del petróleo. También se identificaron fuentes estáticas del conocimiento, como lo fueron los libros de geología y de petróleos, entre otros. Con todo esto entonces se planearon y realizaron diferentes sesiones de elicitación de conocimiento que permitieron identificar los conceptos claves que describen las cuencas de petróleos.

Para desarrollar el modelo se seleccionaron técnicas que ayudaron a identificar conocimiento del tipo conceptual, tales como laddering ${ }^{10}$, clasificación de conceptos ${ }^{11} \mathrm{y}$ algunas otras técnicas de matrices ${ }^{12}$ empleando imágenes que apoyaran los conceptos del modelo.

Siempre el conocimiento elicitado se llevaba a mapas conceptuales para que pudiera ser validado e integrado en el modelo de conocimientos.

Adicionalmente y para facilitar la determinación del alcance del conocimiento de cada una de las 15 cuencas y asegurar la integridad del conocimiento del dominio, se desarrolló un metamodelo que contiene una serie de plantillas del modelo de conocimiento tipo canvas.

Este metamodelo sirve como guía de elaboración de los mapas que describen cada una de las cuencas, incluyendo los conceptos que deben mostrarse a mayor nivel de detalle en los mapas conceptuales. Este metamodelo entonces se desarrolló construyendo la que se denominó como la "Cuenca NN" diseñada como plantilla para que sirviera de base para definir

\footnotetext{
${ }^{10} \mathrm{El}$ experto y el ingeniero construyen conjuntamente una representación gráfica del dominio en términos de las relaciones entre el dominio y la solución de problemas [10].

11 Proceso repetitivo que consiste en hacer ordenamientos de cartas con conceptos con el propósito de hacer agrupaciones por categorización [10].

12 Se emplea para hacer análisis sobre las propiedades de los conceptos. En el caso particular del proyecto se usó empleando imágenes [10].
} 
el mismo contenido para cada una de las cuencas. Está conformado por una colección de 11 mapas plantillas, los cuales son la base para describir cada una de las cuencas. En los mapas se describen diferentes aspectos como la descripción general de la cuenca, mostrando sus características geográficas, estratigráficas y los eventos que conllevaron a su formación, como se mostró en la Figura 5. Además, se tiene una descripción del sistema petrolífero, de los procesos de generación, expulsión y retención y de las características de los hidrocarburos que allí se encuentran. La Figura 6 pertenece a otra plantilla de uno de los mapas que se construyeron para describir las cuencas y que contiene los conceptos principales que conforman el sistema petrolífero.

Como el dominio de la geología es un dominio muy visual, para los geólogos las imágenes son muy ricas en contenido y permiten hacer interpretaciones para las cuales las palabras (conceptos desde un mapa

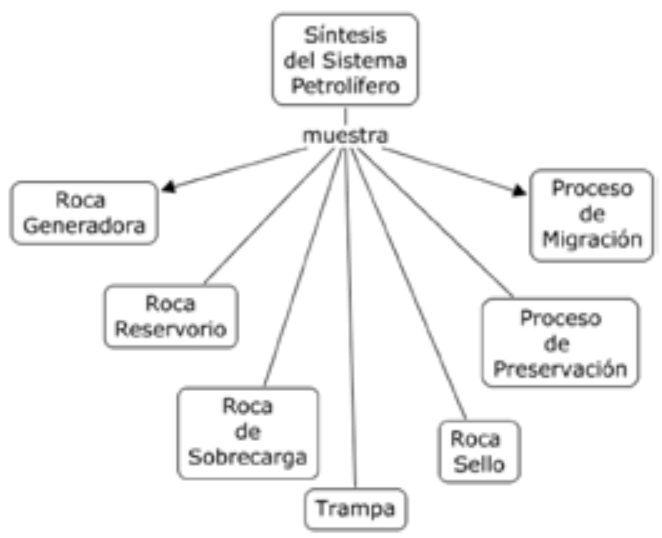

Figura 6. Plantilla para el sistema petrolífero [20].

\section{ANHE}

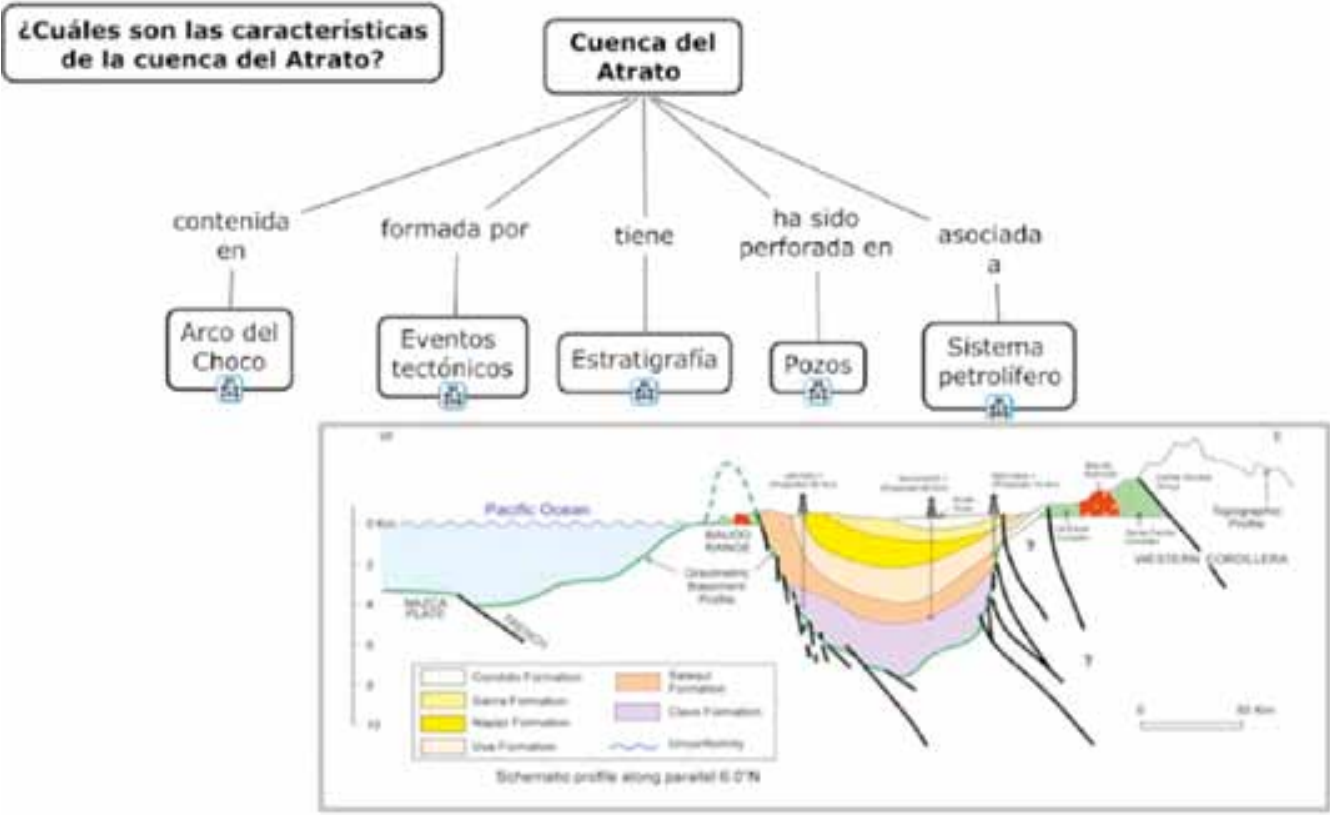

Figura 7. Mapa que muestra un tipo de recurso asociado-mapa geográfico [20-21]. 


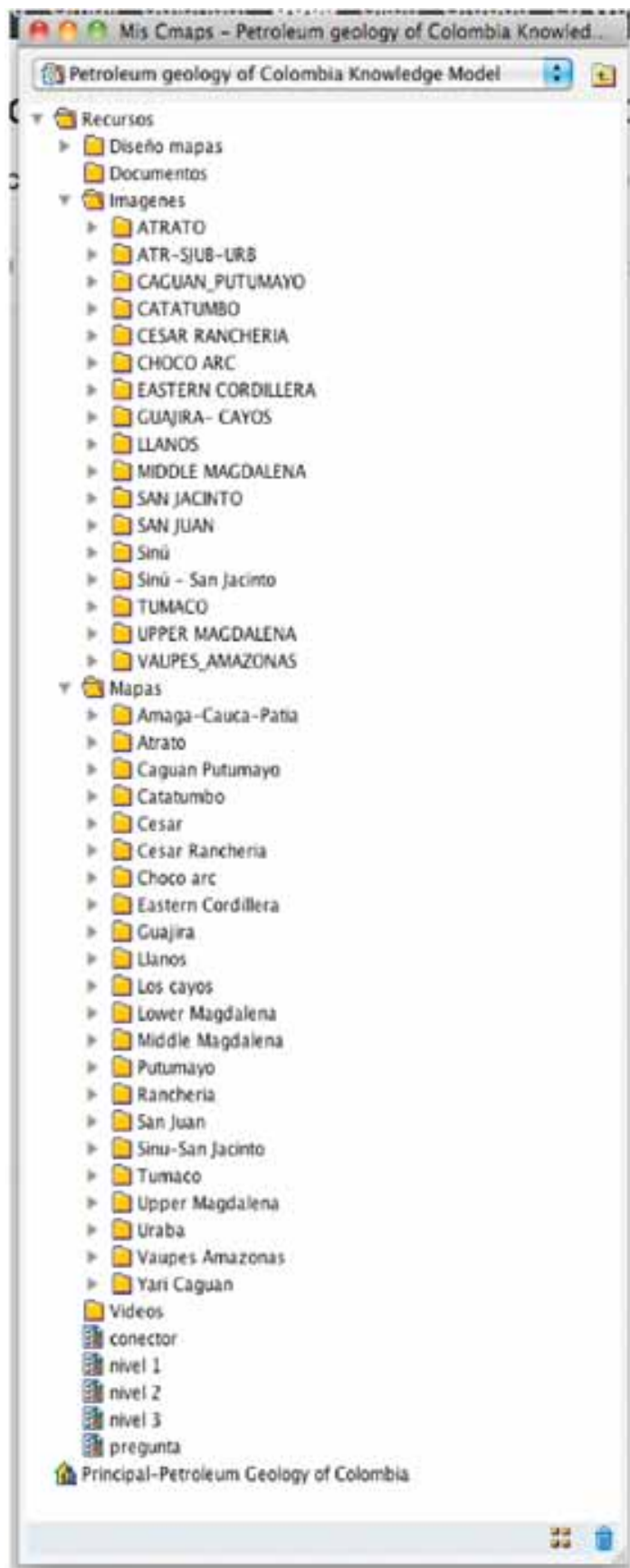

Figura 8. Carpetas imágenes y mapas del modelo de conocimiento [20-21].

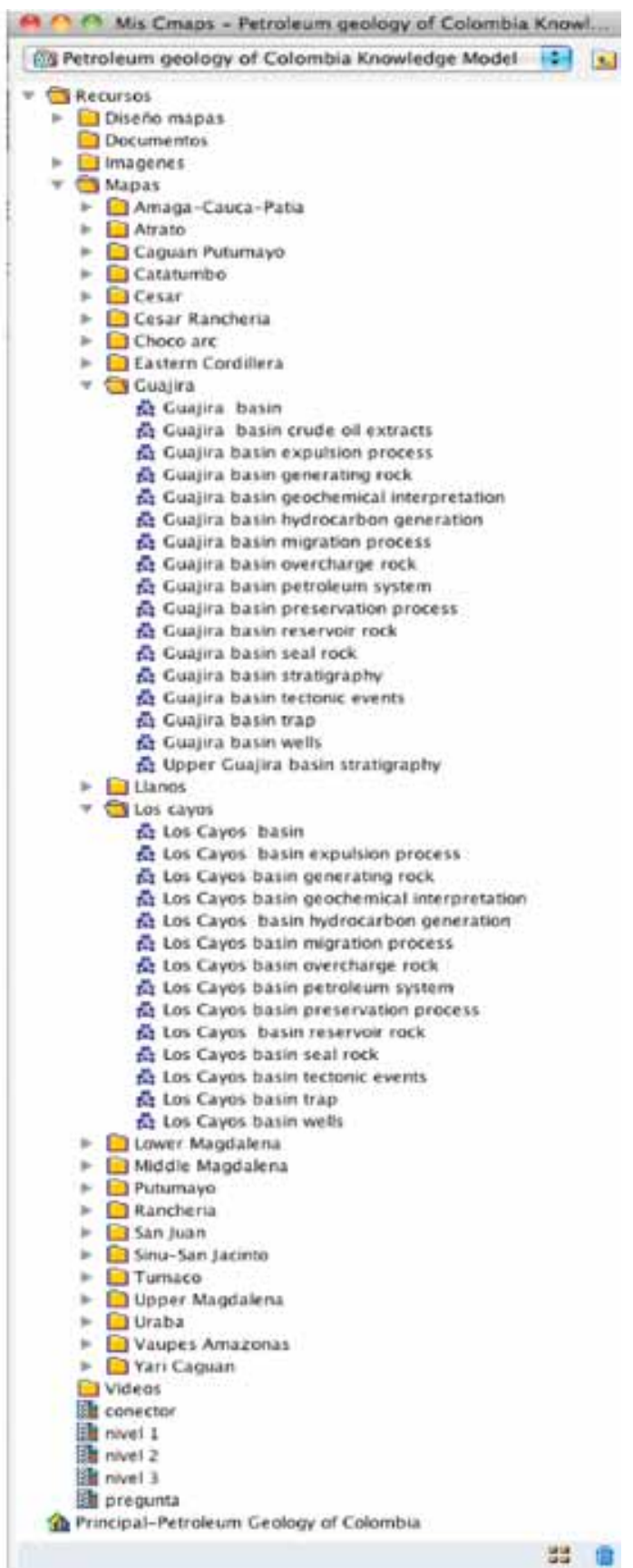

Figura 9. Carpetas del modelo de conocimiento con los mapas asociados [20-21]. 
conceptual) se quedan cortas en nivel de detalles, se definió que el modelo de conocimientos no debía quedarse en una simple colección de mapas conceptuales, sino que tenía que integrar recursos adicionales que apoyaran y reforzaran la gran mayoría de los conceptos. Se incluyeron entonces imágenes de mapas, resultados de pruebas (por ejemplo gravimetría), tablas de información sobre los pozos y videos que reflejaban algún aspecto importante relacionado con la cuenca o con el sistema petrolífero. La Figura 7 corresponde a la vista de un mapa con un recurso tipo imagen que permite ampliar el concepto al que está asociado.

A continuación se presenta la estructura del modelo de conocimiento, organizado por medio de carpetas clasificadas de acuerdo con el tipo de recurso (documentos, mapas, imágenes, videos). Estas figuras permiten evidenciar la magnitud del modelo desarrollado. En la Figura 8 se amplía la carpeta Imágenes y la carpeta Mapas, que contienen la información en subcarpetas de acuerdo con cada una de las cuencas que forman el sistema petrolífero colombiano.

La Figura 9 muestra los contenidos de los mapas que describen las cuencas. Esto permite identificar tanto los mapas básicos creados para cada una de las cuencas como otros mapas que complementan la descripción.

\section{CONCLUSIONES}

Como lo dicen Schreiber y Akkermans y otros en [22] hacer modelos de conocimiento es complicado porque el proceso de modelado en sí es una actividad de solución de problemas constructiva para la que no existe una única "buena" solución. Pero durante el desarrollo del modelo y una vez éste está terminado el conocimiento adquirido es mucho mayor que si se hubiera dejado sin formalizar. Este conocimiento explícito enriquece tanto al que hace el modelo como a todos aquellos que puedan después acceder a él.

Con este resultado, se logra lo que se planteó inicialmente: un Modelo de Conocimiento de cuencas petrolíferas colombianas que apoyará los procesos de unificación, representación y preservación del conocimiento que se tienen de estas cuencas y permitirá la navegación por el conocimiento representados en el modelo, a través de datos y recursos que permiten verlo, tanto en forma global, como con sus relaciones relevantes $\mathrm{y}$ con los formatos apropiados. Esto hace que el conocimiento se vea más dinámico y personalizado, pues dependerá de quien lo consulte, de la forma como lo navegue.

Con este proyecto se puede concluir que la estrategia de modelo de conocimientos apoya el desarrollo de los procesos de la IC, desde la adquisición facilitando la identificación de conceptos relevantes y de las relaciones existentes entre estos con el objeto de poderlos representar adecuadamente hasta la manipulación del conocimiento, para garantizar que el conocimiento corresponde a la realidad del dominio.

La técnica de mapas conceptuales ofrece muchas ventajas para la representación del conocimiento, no sólo por su facilidad de aplicación sino por el soporte formal desde el punto de vista cognitivo que tienen para el conocimiento conceptual para sintetizar y visualizar este conocimiento.

Es importante identificar cuáles son las técnicas y herramientas que pueden apoyar el desarrollo del proyecto de conocimientos, considerando tanto el dominio sobre el cual se trabajará, el producto final a obtener y los expertos con los que se va a trabajar. En el proyecto cuencas petrolíferas colombianas fue necesario identificar que el conocimiento a representar era del tipo conceptual, pero que estaba inmerso en un dominio en el cual las imágenes decían mucho más de lo que las palabras podían representar, por lo que fue necesario tener una representación que uniera ambos aspectos.

Tener una representación visual del conocimiento de un dominio tan complejo y con múltiples conceptos relevantes favorece no únicamente su visualización sino también su navegación.

\section{AGRADECIMIENTOS}

Las autoras quieren dar sus agradecimientos a las ingenieras Andrea Arcila Marín, Juanita Escobar Vélez y Alejandra Cruz Gaviria quienes realizaron las labores de IC y desarrollaron en CmapTools parte el modelo de conocimientos mencionado, dentro de su proyecto de grado de Ingeniería de Sistemas [21]. Además, a los expertos Fabio Cediel Melo y 
Geovanny Bedoya Sanmiguel quienes aportaron todo su conocimiento para que el modelo de conocimientos fuera una realidad. Por último, y no menos importante, agradecemos a la Universidad EAFIT y a la Agencia Nacional de Hidrocarburos por el apoyo recibido al patrocinar parte de este proyecto.

\section{REFERENCIAS}

[1] H. Eriksson and M. Penker. "Business modeling with UML: Business patterns at Work". John Wiley \& Sons, Inc., New York: New York. 2000.

[2] K. Sung-kwan, L. Sengbae and R. Mitchell. "Building A Knowledge Model: A DecisionMaking Approach". Journal of Knowledge Management Practice. August, 2004. URL: http://www.tlainc.com/articl68.htm

[3] A. Vesely. "On the representation of expert procedural knowledge (Reprezentace expertní procedurální znalosti)". Czech University of Agriculture, Prague, Czech Republic. AGRIC. ECON.-CZECH, 52. Vol. 6, pp. 516-521. 2006.

[4] G. Judelman. "Knowledge Visualization; Problems and Principles for Mapping the Knowledge Space". MSc Thesis Dissertation. University of Lübeck. Germany. 2004.

[5] G. Shanks, E. Tansley and R. Weber. "Using Ontology to validate conceptual models". Communications of ACM. Vol. 46, Issue 10, pp. 85-89. 2003.

[6] S. Russell and P. Norving. "Inteligencia Artificial. Un enfoque moderno". Pearson Prentice Hall. Segunda Edición. 2004.

[7] A. Brooking. "Corporate Memory; Strategies for Knowledge Management”. International Thomson Business Press. Londres. 1999.

[8] A. Sánchez. "Una aproximación ontológica al modelado de conocimiento en los dominios de planificación". Tesis doctoral. Universidad Complutense de Madrid. 2010.

[9] M. Filej and D. Hellens. "Knowledge elicitation. A practical handbook". Prentice Hall. UK. 1991.

[10] N. Milton. "Knowledge acquisition in practice". Springer- Verlag. London. 2007.

[11] B. Capon. "Knowledge representation: An approach to artificial intelligence". Academic press Inc. San Diego, CA. 1990.
[12] J. Sánchez. "Sistemas expertos: Una metodología de programación". Macrobit corporation. Miami. 1988.

[13] A. Campos. "Mapas Conceptuales, Mapas mentales y otras formas de representación del conocimiento". Editorial Magisterio. Bogotá. 2005.

[14] R. Davis, H. Shrobe and P. Szolovits. "What is a Knowledge representation". AI Magazine. Vol. 12, Issue 1. 1993.

[15] A. Markman. "Knowledge Representation". Lawrence Erlbaum Associates, Inc. New Jersey. 1999.

[16] A. Cañas, G. Hill and J. Lott. "Support for Constructing Knowledge Models in CmapTools". Technical Report IHMC CmapTools 93-02. 2003. URL: http:// cmapskm.ihmc.us/servlet/SBReadResourc eServlet?rid=1064009710027_142198331 9_27104\&partName=ht mltext

[17] J. Coffey, R. Hoffman, A.J. Cañas and K.M. Ford. "A Concept Map-Based Knowledge Modeling Approach to Expert Knowledge Sharing, IKS 2002". The IASTED International Conference on Information and Knowledge Sharing. Virgin Islands. 2002.

[18] J. Coffey, R. Hoffman and A.J. Cañas. "Concept map-based knowledge modeling: perspective from information to knowledge visualization". Information Visualization. Vol. 5, pp. 192-201. 2006. URL: http://www. palgrave-journals.com/ivs

[19] J. Novak and B. Gowin. "Aprendiendo a aprender”. Ediciones Martínez Roca. España. 1988.

[20] V. Rodríguez. "Diseño e implementación de un modelo de conocimientos sobre las cuencas petrolíferas de Colombia”. Tesis para obtener el grado de Magíster en Ingeniería, Escuela de Ingeniería. Universidad EAFIT. Medellín, Colombia. 2012.

[21] A. Arcila, J. Escobar y A. Cruz. "Portal Web de un modelo de conocimientos de una cuenca petrolífera de Colombia". Proyecto de Grado de Ingeniería de Sistemas. Universidad EAFIT. 2011.

[22] G. Schreiber, H. Akkermans, A. Anjewierden, R. de Hoog. N.R. Shadbolt, W. Van de Velde and B.J. Wielinga. "Engineering of Knowledge and Management; The Common KADS Methodology". The United States of America, The MIT Press, p. 455. 2000. 